\title{
Pediatric Cushing's Syndrome due to primary pigmented nodular adrenocortical disease
}

\author{
Dia eddine Boudiaf*1, Lamia- Sabrina Khesrani ${ }^{2}$, Farida Chentli '. \\ 1 : Department of Endocrinology and Metabolic diseases, Bab El Oved Teaching Hospital, Algiers, Algeria. \\ 2 :Department of pediatric surgery, Mustapha Bacha Hospital, Algiers, Algeria. \\ *boudiaf.dia@outlook.com
}

\section{Introduction:}

Primary pigmented nodular adrenocortical disease (PPNAD) is a rare cause of ACTH-independent Cushing's syndrome in paediatric population. This entity due to genetic mutation has specific histological appearance and can be associated to Carney's complex or to McCune Albright syndrome. Our aim was to report a paediatric case in order to specify clinical and biological characteristics.

\section{Case report:}

A 6 year-old boy with no family or personal medical history was sent for puffiness of the face, stature retardation and progressive weight gain. Clinical examination showed a short stature (-1.5SD) with severe android obesity $\left(\mathrm{BMI}=50.5 \mathrm{~kg} / \mathrm{m}^{2}\right)$, acanthosis nigricans and pubic hair (Tanner stage 3 ), but without any hyper catabolism features. Blood pressure $=100 / 60 \mathrm{mmHg}$. There was not any manifestation of Carney's complex or McCune Albright syndrome. Biological assessment showed endogenous hypercortisolism with low ACTH (ACTH $<01 \mathrm{pg} / \mathrm{ml}$ ) and paradoxical response to dexamethasone. He had bilateral adrenalectomy and histological study pleaded for PPNAD. Research for genetic mutation was negative for PRKAR1A. The follow-up under hydrocortisone and mineralocorticoids' substitution showed an excellent result

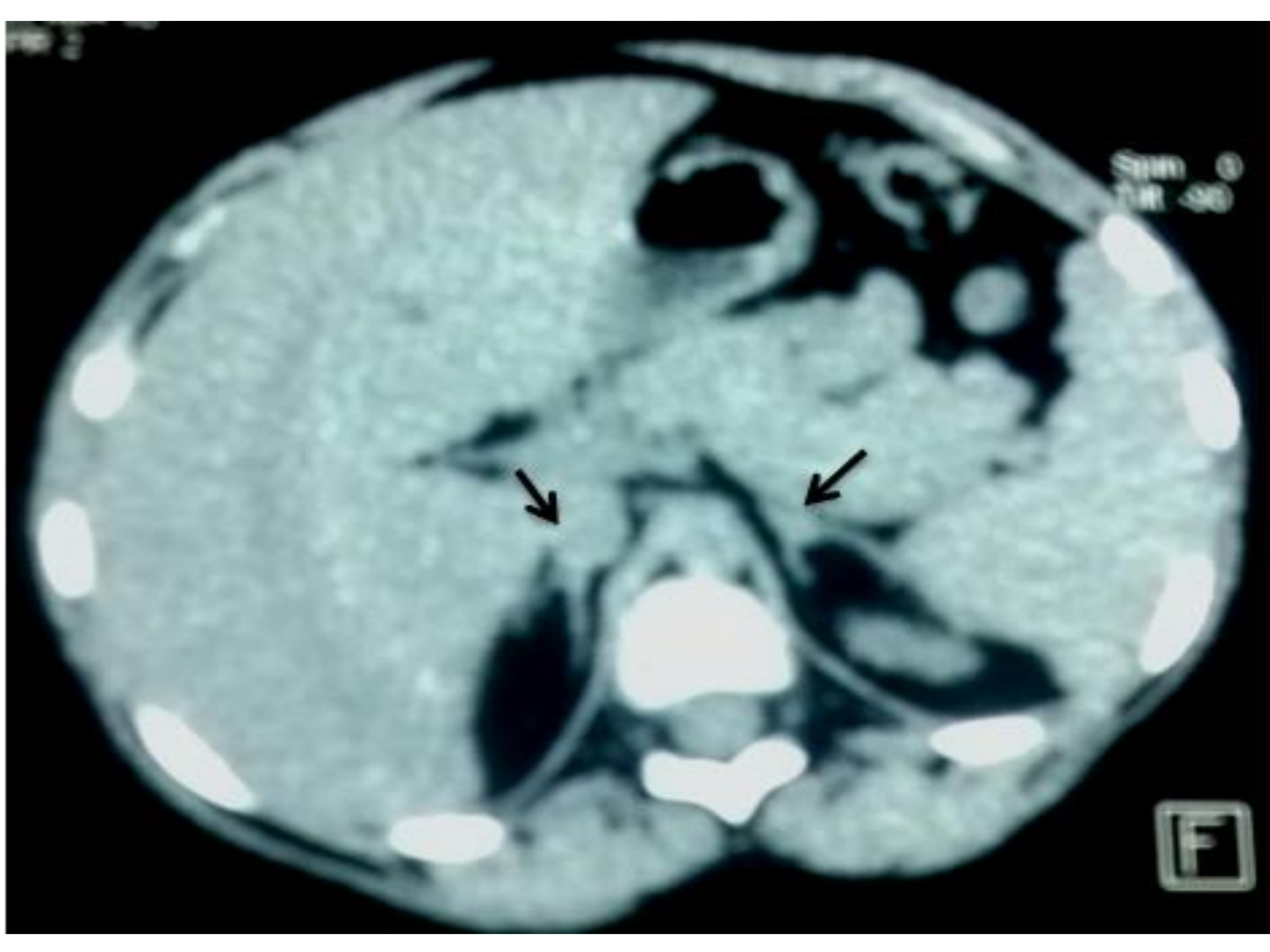

Adrenal glands'

hypertrophy on CT scan before adrenal surgery

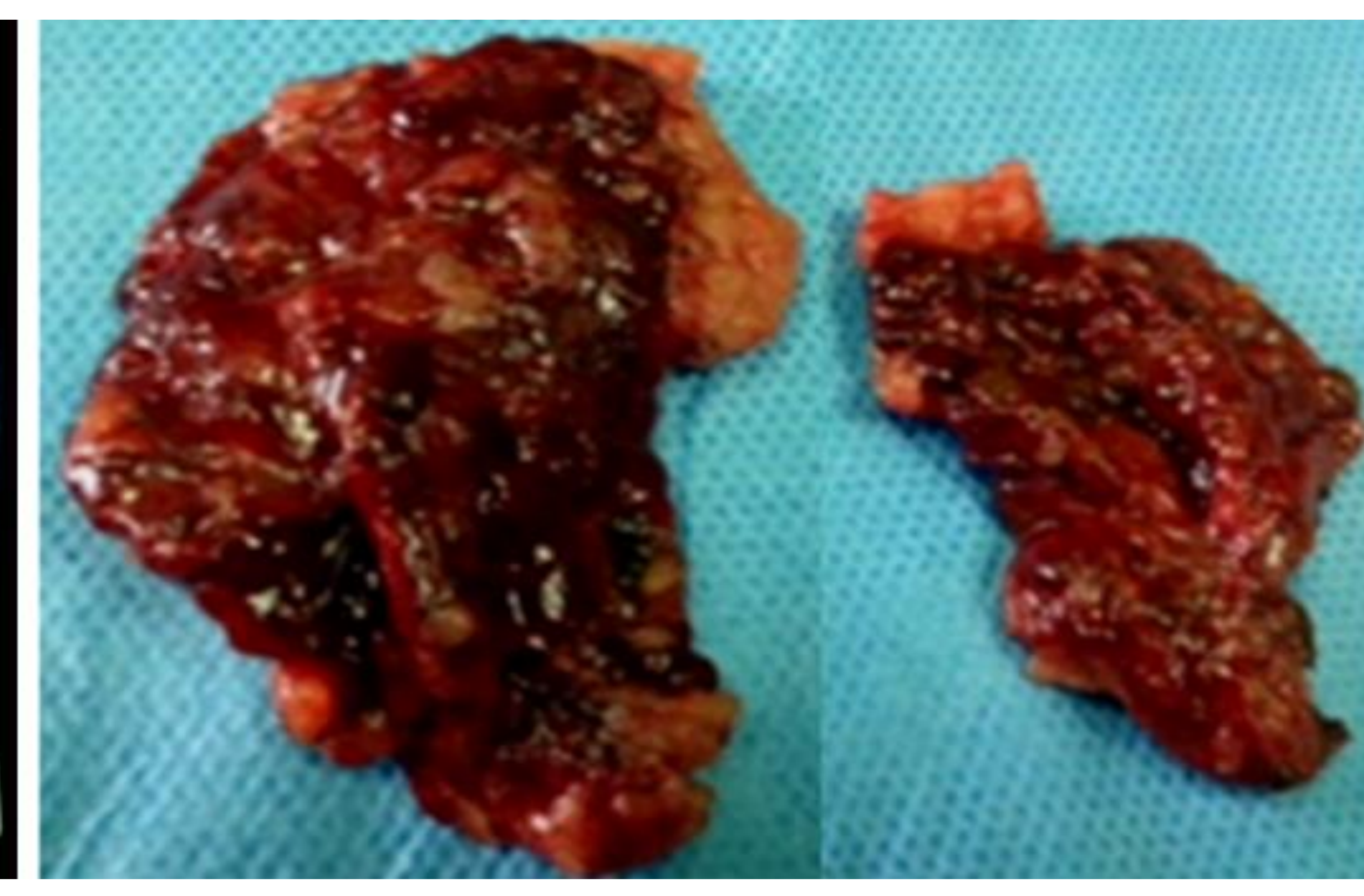

PPNAD anatomical aspect after bilateral adrenalectomy.
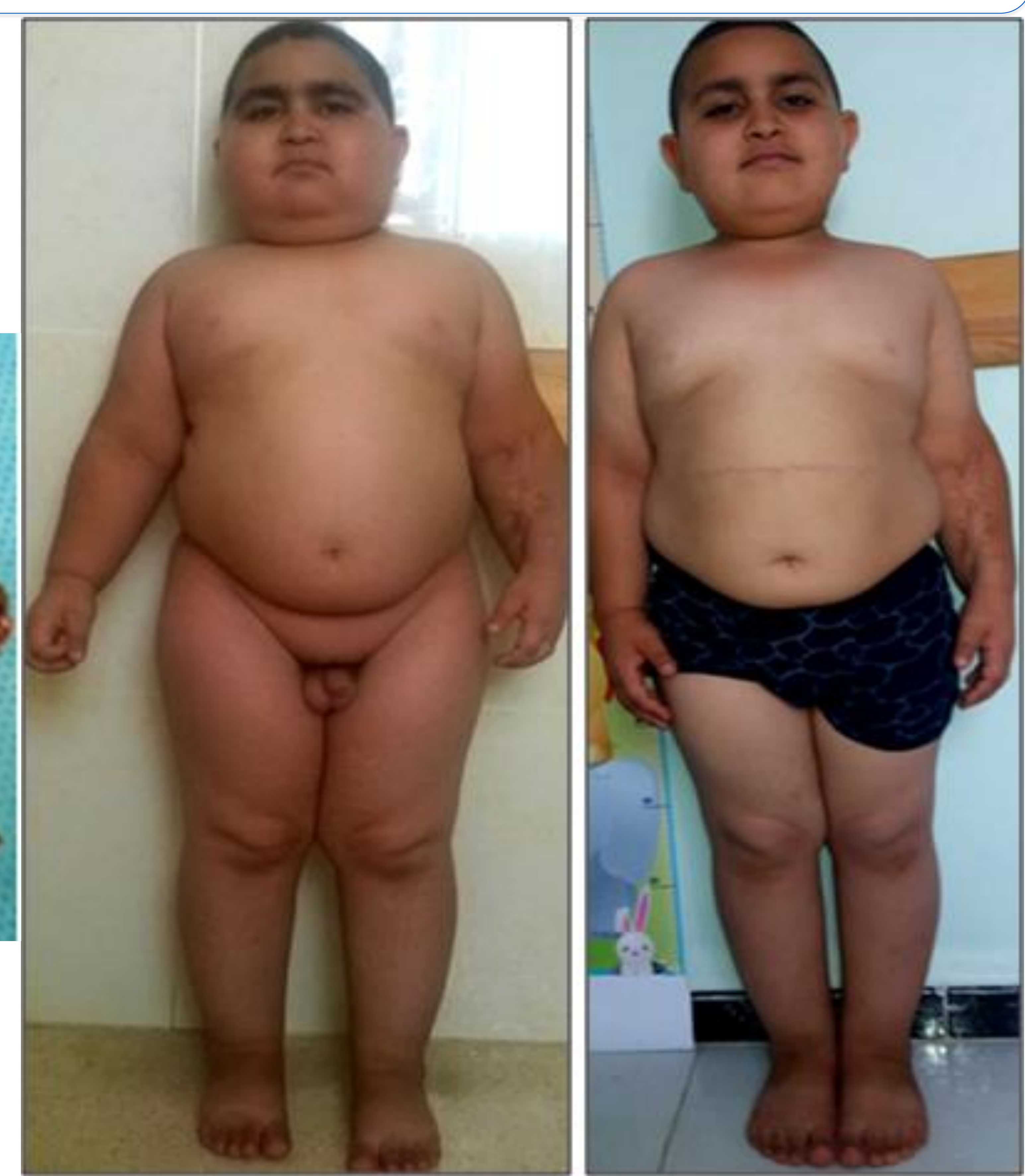

The boy before and after bilateral adrenalectomy (parents' permission to show photos was obtained)

\section{Conclusion:}

PPNAD is a very rare cause of paediatric Cushing syndrome. It may be sporadic or familial. It can also be part of Carney's complex or McCune Albright syndrome which was not the case in our patient. On the biological point this syndrome is characterized by a paradoxical response to dexamethasone as observed in this case. On the physiopathological point, three genetic mutations can cause PPNAD: PRKAR1A, PDE11A, PDE8B. For our patient, research for the most important mutation (PRKAR1A) was negative. The treatment is based on bilateral adrenalectomy to avoid Cushing's numerous morbidities. 